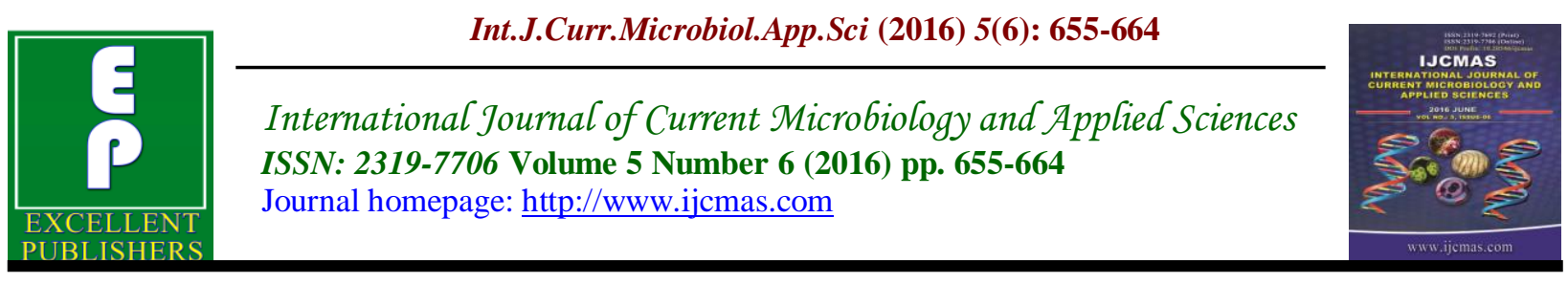

Original Research Article

http://dx.doi.org/10.20546/ijcmas.2016.506.071

\title{
Isolation and Identification of a Bacteriocinic Substance Producing Bacteria from Various Sources
}

\author{
S.B. Ranganath* and T. Sharmila \\ Department of Microbiology and Biotechnology, Jnanabharathi Campus, \\ Bangalore University, Bangalore- 560056, India \\ *Corresponding author
}

\begin{tabular}{|c|c|}
\hline & A B S T R A C T \\
\hline $\begin{array}{l}\text { Bacteriocin, } \\
\text { Gram positive, } \\
\text { Antimicrobial } \\
\text { activity, } \\
\text { pathogens, } \\
\text { isolates. }\end{array}$ & \multirow{3}{*}{$\begin{array}{l}\text { Bacteriocins are low molecular proteinaceous antimicrobial compounds secreted } \\
\text { by bacteria to inhibit the growth of similar or closely related bacterial } \\
\text { strains. In the present study, an attempt was made to isolate bacteria from various } \\
\text { sources and screen for bacteriocinic activity. A total of } 609 \text { bacteria were isolated } \\
\text { from different sources out of which } 303 \text { gram positive bacteria were screened for } \\
\text { antimicrobial activity by primary streak method. Among these, } 51 \text { positive isolates } \\
\text { were studied for colony and cell morphology which exhibited good antimicrobial } \\
\text { activity. Thirty two isolates were streamlined for determination of bacteriocinic } \\
\text { activity by agar well diffusion assay against four pathogens. Further three isolates } \\
\text { IB23, AH4 and BrMk } 4 \text { revealed significantly better antimicrobial activity against } \\
S \text {. aureus, P. aeruginosa and E. coli. very scarce antimicrobial activity was } \\
\text { observed against L. monocytogenes. Based on the biochemical tests, the organism } \\
\text { was identified to be Staphylococcus aureus, Bacillus cereus and Lactobaccilus sp. } \\
\text { respectively. }\end{array}$} \\
\hline Artic & \\
\hline $\begin{array}{l}\text { Accepted: } \\
\text { 23 May } 2016 \\
\text { Available Online: } \\
\text { 10 June } 2016\end{array}$ & \\
\hline
\end{tabular}

\section{Introduction}

Bacteriocins are generally ribosomally synthesised low molecular weight antimicrobial peptides that are not lethal to the host cells (Law, 2005). It was first discovered by Gratia in 1925 (Gratia, 2000) and they find their application in gastrointestinal disorders, biotechnological, food and agro-industries (Jack et al., 1995). The antimicrobial effect of bacteriocins (Sahu et al., 2008) have a potential use as natural food preservatives (Riley, 2009). Prevention of spoilage and pathogenic microorganisms will be more efficient if bacteriocinic activity is increased. Bacteriocins of Gram- positive bacteria are abundantly found and are more diverse than those found in Gram negative bacteria and they resemble many of the antimicrobial peptides produced by eukaryotes. They are generally non lethal cationic, amphiphilic and membranepermeabilizing peptides (Sahl et al., 1998). They can provide both broad-spectrum killing of many microbes or can target on individual bacterial species.

Besides these applications, they can also be used in combination with other antibiotics for therapeutic use which finds $\$ 22$ to $\$ 24$ 
billion globally at $2-3 \%$ increase per annum (Malini Maria, 2012). Their use in food and dairy industry lies in preservation by increasing the shelf- life of the products and are also used as additives, flavour enhancers which replaces harmful chemicals. They also find their application in livestock by feeding on bacteriocin producing bacteria and as probiotics in aquaculture.

\section{Materials and Methods}

\section{Isolation of the Culture}

A total of 51 different samples, as shown in table 1, were collected in sterile containers. All samples were appropriately labelled and transported to the lab and stored at $10^{\circ} \mathrm{C}$ for further investigations. Different procedures were followed for different samples based on the load of the microbes as shown in table 1. Serial dilutions of the collected sample were carried out and $0.1 \mathrm{ml}$ of each diluents was transferred to $0.9 \mathrm{ml}$ of sterile distilled water.

Correspondingly dilutions were prepared from $10^{-2}$ to $10^{-4}$ according to the sample. The dilution of $10^{-3}$ and $10^{-4}$ were used for isolation of bacteria. The inoculum was spread on Nutrient agar (NA) plates and the plates were incubated at $37^{\circ} \mathrm{C}$ for 24 hours. Morphological appearances of the inoculated plates were observed after 24 hours of incubation (Fig 1.) and distinct colonies were sub-cultured to obtain pure isolates which were then subcultured on NA slants.

The pure bacterial isolates (Fig 2.) were further identified by microscopic examination after Gram staining. The Colonies which appeared as purple blue i.e. Gram positive upon microscopic observations were subcultured on Nutrient agar plates and preserved at $4^{\circ} \mathrm{C}$ in the refrigerator for further experiments.

\section{Preliminary Screening of Antimicrobial activity}

The pure isolates were further screened for bacteriocinic activity by single streak method against 4 pathogenic bacteria procured from Culture collection centers (Escherichia coli ATCC 25922, Pseudomonas aeruginosa ATCC- 27853, Staphylococcus aureus ATCC 13709, and Listeria monocytogenes MTCC 1143) as shown in Table 2. Mueller Hinton Agar medium was prepared and aseptically poured into Petriplates. After solidification, lawn culture of indicator microorganisms were made on the agar surface by using sterile cotton swabs. The plates were incubated for 15 minutes in room temperature inside the laminar air flow. After incubation the isolated cultures were streaked in single line using a sterile inoculating loop. The plates were incubated at $37^{\circ} \mathrm{C}$ for 24 hours (Fig 3.). After 24 hours of incubation period, microorganisms displaying clear zones of inhibition against the pathogens were observed.

\section{Secondary Screening of Antimicrobial Activity}

Antimicrobial activity was analysed against various pathogens mentioned above by agar well diffusion method. The pathogens were incubated in Brain-Heart infusion broth at $37^{\circ} \mathrm{C}$ for different hours and adjusted according to $0.5 \mathrm{McFarland}$ standards was used. The pathogens were lawn cultured using sterile cotton swab. Agar well was made using sterile cork borer and $50 \mu \mathrm{l}$ of isolate supernatants grown in MRS media (centrifuged at 10,000 $\mathrm{g}$ for 15 minutes) was incorporated into the wells and incubated for 24 hours at $37^{\circ} \mathrm{C}$. After incubation, zone of inhibition was measured (in $\mathrm{mm}$ ) as shown in table 2. (Fig 4.). 
Table.1 Cultural characters of the isolated bacterial colonies from various sources

\begin{tabular}{|c|c|c|c|c|c|c|c|c|c|c|}
\hline $\begin{array}{l}\text { SI } \\
\text {.No. }\end{array}$ & Samples & Dilu-tion & $\begin{array}{l}\text { No of } \\
\text { Colonies }\end{array}$ & $\begin{array}{l}\text { Form/ } \\
\text { Shape }\end{array}$ & Size & Elevation & $\begin{array}{l}\text { Surface/ } \\
\text { Texture }\end{array}$ & Margin & Colour & Gram Stain \\
\hline 1 & Curd nandini & $10^{-3}$ & 11 & $\begin{array}{l}\text { Spreading, } \\
\text { Irregular }\end{array}$ & Large & Umbonate & Dry & Undulate & $\begin{array}{l}\text { Creamy } \\
\text { Off white }\end{array}$ & Positive rod \\
\hline 2 & Curd neighbor & $10^{-3}$ & 22 & Irregular & Moderate & Umbonate & Rough & Wavy & white & Positive rod \\
\hline 3 & Curd NDRI & $10^{-3}$ & 15 & Irregular & Moderate & Convex & dull & Undulate & off white & Positive rod \\
\hline 4 & Curd Tirumala & $10^{-3}$ & 24 & Irregular & Moderate & Flat & Dry & Lobate & White & Positive rod \\
\hline 5 & Curd nestle & $10^{-4}$ & 7 & Circular & Small & Umbonate & Rough & Undulate & $\begin{array}{l}\text { Creamy / } \\
\text { offwhite }\end{array}$ & Positive rod \\
\hline 6 & Curd nilgiris & $10^{-4}$ & 9 & Filamentous & Large & Flat & Rough & Undulate & white & Positive rod \\
\hline 7 & Curd Amul & $10^{-4}$ & 7 & Irregular & Moderate & Convex & dull & Undulate & Cream & Positive rod \\
\hline 8 & $\begin{array}{l}\text { Idly Batter sagar } \\
\text { hotel }\end{array}$ & $10^{-3}$ & 14 & Circular & Small & Flat & Rough & Wavy & $\begin{array}{l}\text { Creamy / off } \\
\text { white }\end{array}$ & Positive rod \\
\hline 9 & $\begin{array}{l}\text { Idly Batter } \\
\text { Srinivas } \\
\text { Darshini }\end{array}$ & $10^{-3}$ & 12 & Irregular & Moderate & Flat & Rough & Undulate & White & Positive rod \\
\hline 10 & $\begin{array}{l}\text { Idly Batter megha } \\
\text { Sagar }\end{array}$ & $10^{-3}$ & 22 & Circular & Small & Flat & Dry & Lobate & $\begin{array}{l}\text { Creamy } \\
\text { Off white }\end{array}$ & Positive rod \\
\hline 11 & Idly B & $10^{-3}$ & 22 & $\begin{array}{l}\text { Spreading, } \\
\text { Irregular }\end{array}$ & Large & Umbonate & dull & Undulate & off white & Positive rod \\
\hline 12 & Idly Batter & $10^{-4}$ & 15 & Irregular & Moderate & Umbonate & dull & Undulate & off white & Positive rod \\
\hline 13 & Idly Batter suma & $10^{-4}$ & 13 & Circular & Small & Flat & Rough & Undulate & $\begin{array}{l}\text { Creamy } \\
\text { Off white }\end{array}$ & Positive rod \\
\hline 14 & Raw Milk & $10^{-3}$ & 3 & Irregular & Moderate & Flat & Rough & Wavy & Whit & Positive rod \\
\hline 15 & Raw Milk & $10^{-3}$ & 5 & Irregular & Moderate & Umbonate & Rough & Lobate & whie & Positive rod \\
\hline 16 & Raw Milk & $10^{-3}$ & 3 & Filamentous & Moderate & Umbonate & Dry & Undulate & whte & Positive rod \\
\hline 17 & Raw Milk & $10^{-3}$ & 3 & \begin{tabular}{|l} 
Spreading, \\
Irregular
\end{tabular} & Large & Convex & dull & Wavy & off white & Positive rod \\
\hline 18 & Breast Milk & $10^{-3}$ & 5 & Circular & Small & Flat & Rough & Undulate & $\begin{array}{l}\text { Creamy } \\
\text { Off white }\end{array}$ & Positive rod \\
\hline 19 & Breast Milk & $10^{-3}$ & 1 & $\begin{array}{l}\text { Spreading, } \\
\text { Irregular } \\
\end{array}$ & Large & Umbonate & Dry & Lobate & $\begin{array}{l}\text { Creamy } \\
\text { Off white }\end{array}$ & Positive rod \\
\hline 20 & Breast Milk & $10^{-4}$ & 5 & Filamentous & Large & Umbonate & Rough & Undulate & white & Positive rod \\
\hline
\end{tabular}




\begin{tabular}{|c|c|c|c|c|c|c|c|c|c|c|}
\hline 21 & Breast Milk & $10^{-4}$ & 2 & Irregular & Moderate & Convex & dull & Undulate & Cream & Positive rod \\
\hline 22 & Breast Milk 1 & $10^{-4}$ & 2 & Circular & Small & Umbonate & Rough & Undulate & $\begin{array}{l}\text { Creamy } \\
\text { Off white }\end{array}$ & Positive rod \\
\hline 23 & Buttermilk 1 & $10^{-3}$ & 11 & Irregular & Small & Flat & Rough & Lobate & white & Positive rod \\
\hline 24 & Buttermilk 2 & $10^{-3}$ & 09 & Irregular & Moderate & Flat & Rough & Undulate & white & Positive rod \\
\hline 25 & Buttermilk 3 & $10^{-3}$ & 05 & Irregular & Moderate & Umbonate & Dry & Undulate & White & Positive rod \\
\hline 26 & Buttermilk 4 & $10^{-3}$ & 23 & Irregular & Moderate & Convex & Rough & Wavy & $\begin{array}{l}\text { Yellowish } \\
\text { white }\end{array}$ & Positive rod \\
\hline 27 & Buttermilk 5 & $10^{-3}$ & 27 & Irregular & Moderate & Umbonate & Rough & Undulate & white & Positive rod \\
\hline 28 & Buttermilk 6 & $10^{-4}$ & 21 & Filamentous & Large & Flat & Rough & Undulate & white & Positive rod \\
\hline 29 & Buttermilk 7 & $10^{-4}$ & 19 & $\begin{array}{l}\text { Spreading, } \\
\text { Irregular }\end{array}$ & Moderate & Convex & dull & Undulate & $\begin{array}{l}\text { Creamy } \\
\text { Off white }\end{array}$ & Positive rod \\
\hline 30 & Buttermilk 8 & $10^{-4}$ & 21 & Circular & Moderate & Flat & Rough & Undulate & $\begin{array}{l}\text { Creamy } \\
\text { Off white }\end{array}$ & Positive rod \\
\hline 31 & Infant faeces 1 & $10^{-3}$ & 5 & Irregular & Moderate & Umbonate & Dry & Wavy & Cream & Positive rod \\
\hline 32 & Infant faeces 2 & $10^{-3}$ & 6 & Irregular & Moderate & Convex & dull & Undulate & Cream & Positive rod \\
\hline 33 & Infant faeces 3 & $10^{-3}$ & 3 & Irregular & Moderate & Umbonate & dull & Undulate & off white & Positive rod \\
\hline 34 & Infant faeces 4 & $10^{-3}$ & 4 & Circular & Small & Flat & Rough & Undulate & $\begin{array}{l}\text { Creamy } \\
\text { Off white }\end{array}$ & Positive rod \\
\hline 35 & Infant faeces 5 & $10^{-4}$ & 5 & $\begin{array}{l}\text { Spreading, } \\
\text { Irregular }\end{array}$ & Large & Umbonate & Dry & Undulate & $\begin{array}{l}\text { Creamy } \\
\text { Off white }\end{array}$ & Positive rod \\
\hline 36 & Infant faeces 6 & $10^{-4}$ & 6 & Irregular & Moderate & Flat & Rough & Wavy & white & Positive rod \\
\hline 37 & Lassi & $10^{-4}$ & 7 & Irregular & Moderate & Flat & Dry & Undulate & white & Positive rod \\
\hline 38 & Yogurt & $10^{-3}$ & 8 & Irregular & Moderate & Umbonate & Rough & Lobate & white & Positive rod \\
\hline 39 & Yogurt & $10^{-3}$ & 9 & Irregular & Moderate & Convex & dull & Lobate & off white & Positive rod \\
\hline 40 & Ant hill soil 1 & $10^{-3}$ & 10 & Circular & Small & Flat & Dry & Undulate & $\begin{array}{l}\text { Creamy } \\
\text { Off white }\end{array}$ & Positive rod \\
\hline 41 & Ant hill soil 2 & $10^{-3}$ & 21 & Filamentous & Large & Flat & Rough & Lobate & Whit & Positive rod \\
\hline 42 & Ant hill soil 3 & $10^{-4}$ & 19 & $\begin{array}{l}\text { Spreading, } \\
\text { Irregular }\end{array}$ & Large & Umbonate & Dry & Undulate & $\begin{array}{l}\text { Creamy } \\
\text { Off white }\end{array}$ & Positive rod \\
\hline 43 & Ant hill soil 4 & $10^{-4}$ & 18 & Irregular & Moderate & Umbonate & Rough & Wavy & white & Positive rod \\
\hline 44 & $\begin{array}{l}\text { Dosa batter } \\
\text { neighbour } 1\end{array}$ & $10^{-4}$ & 15 & Irregular & Moderate & Umbonate & Rough & Lobate & white & Positive rod \\
\hline 45 & Dosa batter & $10^{-4}$ & 32 & Irregular & Moderate & Convex & dull & Lobate & off white & Positive rod \\
\hline
\end{tabular}


Int.J.Curr.Microbiol.App.Sci (2016) 5(6): 655-664

\begin{tabular}{|l|l|l|l|l|l|l|l|l|l|}
\hline & neighbour 2 & & & & & & & & \\
\hline 46 & $\begin{array}{l}\text { Dosa batter } \\
\text { neighbour 3 }\end{array}$ & $10^{-4}$ & 13 & Circular & Small & Flat & Dry & Undulate & $\begin{array}{l}\text { Creamy } \\
\text { Off white }\end{array}$ \\
\hline 47 & $\begin{array}{l}\text { Dosa batter } \\
\text { neighbour 4 }\end{array}$ & $10^{-4}$ & 23 & Filamentous & Large & Flat & Rough & Lobate & White \\
\hline 48 & $\begin{array}{l}\text { Dosa batter } \\
\text { neighbour 5 }\end{array}$ & $10^{-4}$ & 15 & $\begin{array}{l}\text { Spreading, } \\
\text { Irregular }\end{array}$ & Large & Umbonate & Dry & Undulate & $\begin{array}{l}\text { Creamy } \\
\text { Off white }\end{array}$ \\
\hline 49 & $\begin{array}{l}\text { Dosa batter sagar } \\
\text { Hotel }\end{array}$ & $10^{-4}$ & 24 & Irregular & Moderate & Umbonate & Rough & Wavy & Positive rod \\
\hline 50 & $\begin{array}{l}\text { Fruit juice sample } \\
\text { white }\end{array}$ & $10^{-4}$ & 5 & Irregular & Moderate & Convex & dull & Lobate & Positive rod \\
\hline 51 & $\begin{array}{l}\text { Fruit juice sample } \\
2\end{array}$ & $10^{-4}$ & 4 & Irregular & Moderate & Umbonate & Rough & Lobate & Positive rod \\
\hline
\end{tabular}


Table.2 Antimicrobial activity of isolated bacteria (zone of inhibition in $\mathrm{mm}$ )

\begin{tabular}{|c|c|c|c|c|}
\hline Sl..No. & Organism code & S. aureus & E. coli & L. monocytogenes \\
\hline 1. & $\mathrm{CN} 4$ & 5 & 6 & 4 \\
\hline 2 & $\mathrm{CN} 5$ & 6 & 5 & 4 \\
\hline 3. & C T 5 & 6 & 7 & 4 \\
\hline 4. & IB 23 & 9 & 7 & 4 \\
\hline 5. & IB 5 & 7 & 6 & 4 \\
\hline 6. & BM 4 & 6 & 4 & 4 \\
\hline 7. & BM 20 & 4 & 7 & 4 \\
\hline 8. & BM 22 & 6 & 7 & 4 \\
\hline 9. & BM 24 & 6 & 7 & 4 \\
\hline 10. & AH 4 & 8 & 8 & 5 \\
\hline 11. & $\mathrm{AH} 7$ & 7 & 5 & 4 \\
\hline 12. & $\mathrm{AH} 8$ & 7 & 4 & 4 \\
\hline 13. & AH 9 & 6 & 7 & 4 \\
\hline 14. & AH 12 & 7 & 6 & 4 \\
\hline 15. & BrMk 3 & 7 & 6 & 4 \\
\hline 16. & BrMk 4 & 9 & 9 & 5 \\
\hline 17. & BrMk 5 & 5 & 7 & 4 \\
\hline 18. & BrMk 6 & 4 & 7 & 4 \\
\hline 19. & DB S & 7 & 4 & 4 \\
\hline 20. & DB 5 & 5 & 8 & 4 \\
\hline 21. & DB 6 & 6 & 7 & 4 \\
\hline 22. & DB 7 & 5 & 4 & 4 \\
\hline 23. & DB 8 & 8 & 5 & 4 \\
\hline 24. & YG 3 & 4 & 6 & 4 \\
\hline 25. & YG 4 & 5 & 5 & 4 \\
\hline 26. & $\mathrm{Fj} 2$ & 4 & 5 & 4 \\
\hline 27. & $\mathrm{Fj} 3$ & 4 & 6 & 4 \\
\hline 28. & RM 2 & 5 & 4 & 4 \\
\hline 29. & RM 3 & 6 & 4 & 4 \\
\hline 30. & RM 4 & 5 & 4 & 4 \\
\hline 31. & RM 5 & 5 & 5 & 4 \\
\hline 32. & RM 6 & 6 & 5 & 4 \\
\hline
\end{tabular}


Table.3 Biochemical tests of potential isolates

\begin{tabular}{|l|l|l|l|}
\hline Biochemical test & IB23 & AH4 & BrMk 4 \\
\hline Indole & - & - & - \\
\hline MR & + & - & + \\
\hline VP & + & - & - \\
\hline Citrate & + & + & - \\
\hline Catalase & + & + & - \\
\hline Starch & + & - & + \\
\hline Urease & - & - & - \\
\hline Nitrate reduction & - & - & - \\
\hline Casein hydrolysis & - & - & - \\
\hline Glucose fermentation & + & + & $+\mathrm{A} / \mathrm{G}$ \\
\hline Fructose Fermentation & + & $+/ \mathrm{A}$ & + \\
\hline Mannose Fermentation & + & $+/ \mathrm{A}$ & + \\
\hline Galactose Fermentation & - & $+/ \mathrm{A}$ & + \\
\hline Lactose fermentation & + & - & + \\
\hline Gelatin hydrolysis & + & - & - \\
\hline H 2 S Production & + & - & - \\
\hline Oxidase & - & + & - \\
\hline
\end{tabular}

Fig.1 Isolation of bacteria from various samples

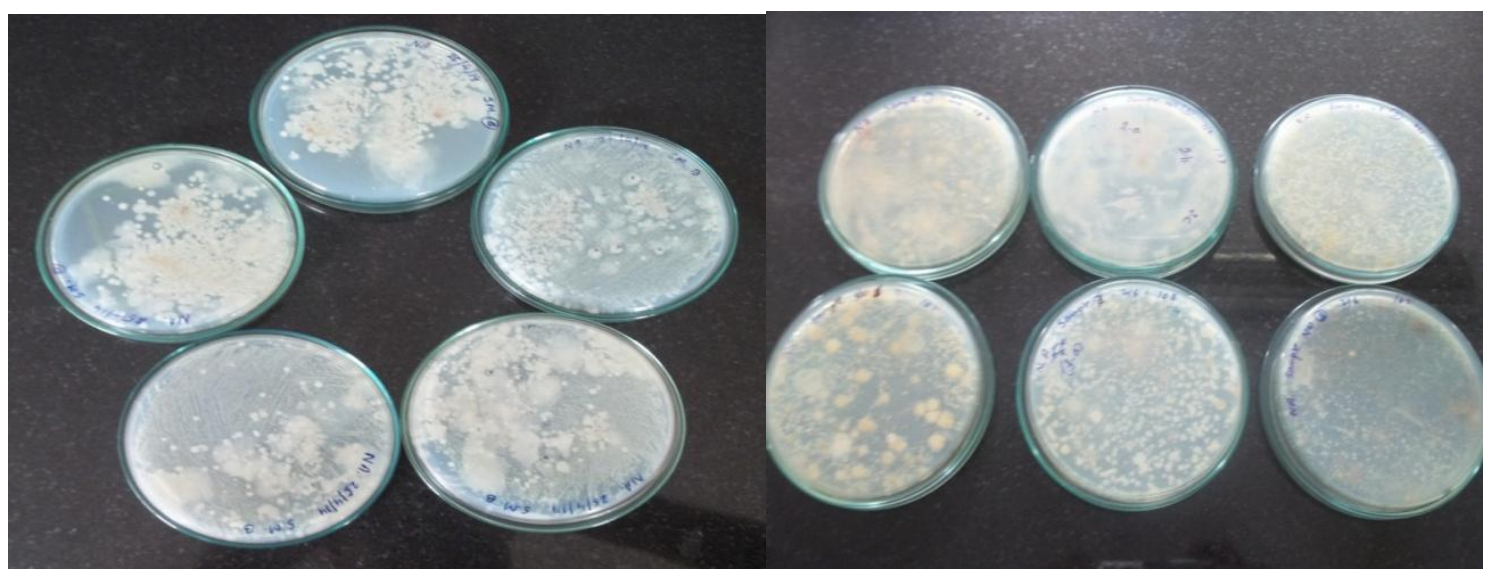


Fig.2 Isolated pure culture

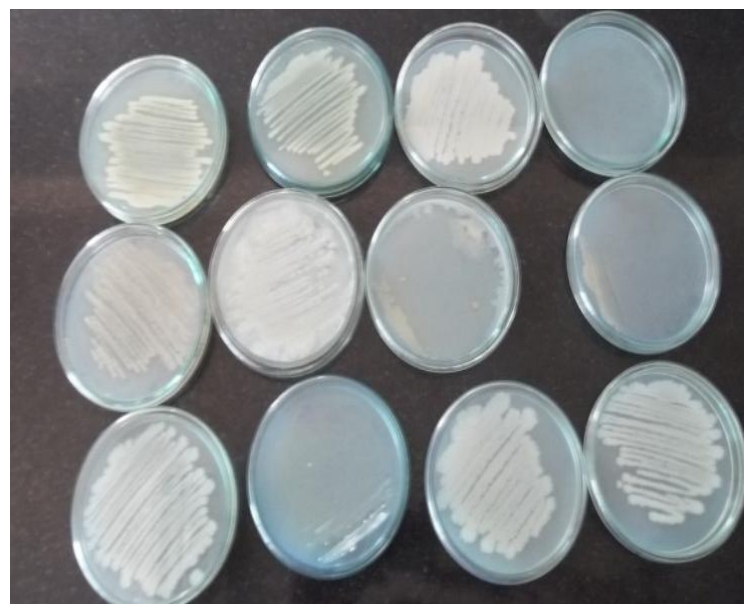

Fig.3 Initial screening of the isolates against a few pathogens
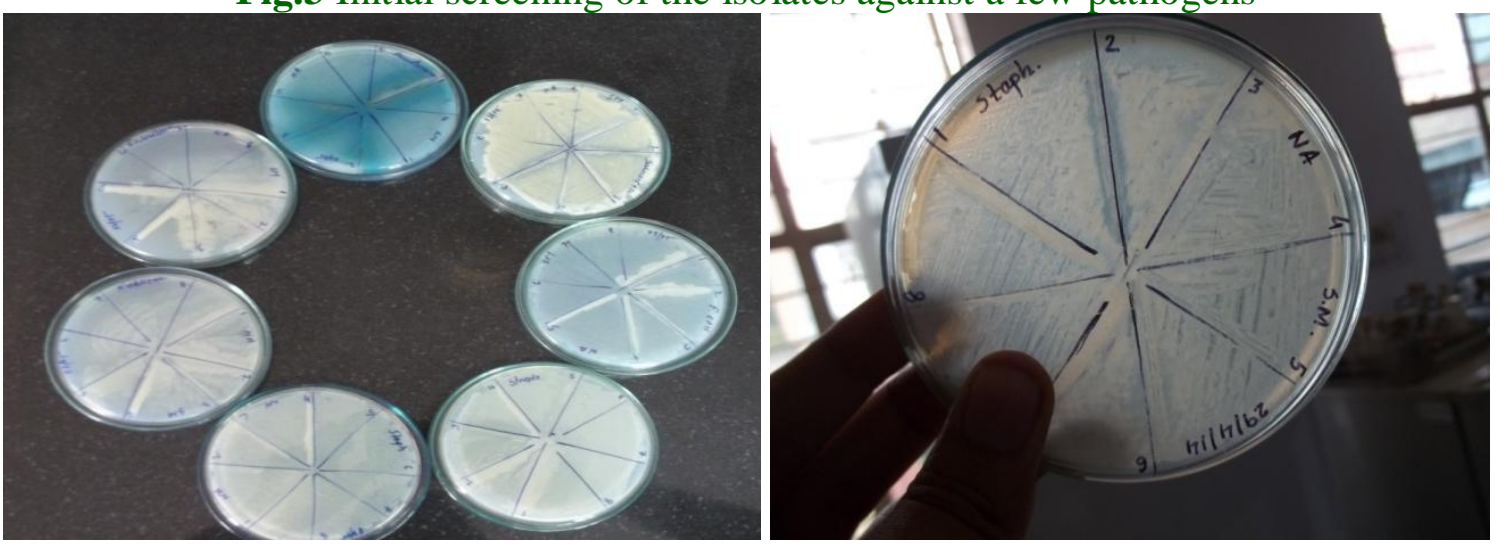

Fig.4 Antimicrobial activity of the isolates

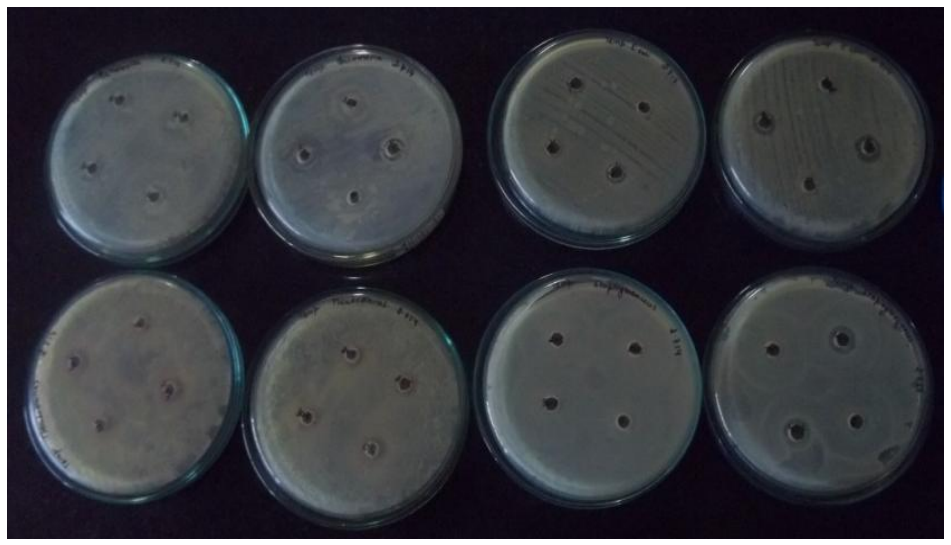

\section{Biochemical Tests}

Different Biochemical tests have been performed for identification of potential isolates., (Table 3) according to Bergey's Manual of Bacteriology like indole test, methyl Red test, Vogue's Proskeur's test, citrate test, catalase test, starch, urease, 
nitrate reduction test, casein hydrolysis, glucose fermentation test, fructose fermentation test, mannose fermentation test, galactose fermentation test, oxidase test, lactose fermentation test, gelatin hydrolysis test and $\mathrm{H}_{2} \mathrm{~S}$ production test.

\section{Results and Discussion}

A total of 609 bacteria were isolated from various sources, among them 303 gram positive bacteria were screened for antimicrobial activity by primary streak method. Out of these, 51 positive isolates were studied for colony morphology (colour, shape, margin, elevation and surface) and cell morphology (shape, arrangement, and Gram's staining) which exhibited good antimicrobial activity. Further, after secondary screening, 32 positive isolates were selected as they exhibited zone of inhibition against all the four pathogens as shown in the Table 2. On secondary screening three isolates IB23, AH4 and BrMk 4 revealed significantly better antimicrobial activity against $S$. aureus, $P$. aeruginosa and E. coli. However, very scarce antimicrobial activity was observed against L. monocytogenes. According to the biochemical tests as shown in Table 3 and the microscopic observation revealed IB23 as $S$. aureus, the organism AH 4 was Bacillus cereus and BrMk 4 belonged to Lactobaccilus sp.

Ashok et al., 2014 reported better antimicrobial activity of isolates from milk and curds against $S$.aureus, $K$.pneumoniae E.coli, however $P$. aeruginosa was resistant against these isolates. In the present study 32 isolates showed activity against $P$. aeruginosa. Similar to the bacteriocinic activity from Lactobacillus sp. isolated from breast milk, Abdulla et al., 2014, also quoted antimicrobial compounds from Lactobacillus acidophilus. Our study on antimicrobial activity of Lactobacillus sp. were similar to the reports of L. plantarum of Das et al., 2014. However Gaamouche et al., 2014, reported better antimicrobial activity of lactic acid bacteria against Listeria monocytogenes.

Bacteriocins of Gram-positive bacteria are abundant and diverse than that of Gram negative bacteria and also differ in ecological and evolutionary aspects. The spectrum of antimicrobial activity is broader in gram-positive bacteria, than that of gram negative species and also less toxic for preservation. In addition, the gram-positive bacteria have relatively higher-molecularweight, heat-labile bacteriocin-like substances.

In conclusion, the present study on isolation and identification of a bacteriocinic substance producing organisms from various sources provides an overview of the diversity of the ability of microbes to produce antimicrobial substances which could be commercially exploited by the food, dairy, medical industries. It is also evident that the bacteriocin like products of gram-positive bacteria revealed broader antibacterial spectrum and will continue to be an active area of applied research.

\section{References}

Abdulla. A. 2014. Antimicrobial Activity of Lactobacillus acidophilus that carry the Bacteriocin Gene. Int. J. Curr. Microbiol. App. Sci., 3(6): 269-276.

Ashok, V., Gomashe, Anjali, A., Sharma, Mamta, A., Wankhede. 2014. Screening and Evaluation of Antibacterial Activity of Bacteriocin Producing Lab against some Selected Bacteria Causing Food Spoilage. Int. 
J. Curr. Microbiol. App. Sci., 3(8): 658-665.

Bemena., L., Mohamed. L., Fernandes, A. M., and. Lee., B H. 2014. Applications of bacteriocins in food, livestock health and medicine. Int. J. Curr. Microbiol. App. Sci., 3(12): 924-949

Das. A., Sasidharan, S., Achuthan, T., Sindhuja, M.E. 2014. Isolation, Characterization and Estimation of Antimicrobial Activity of Novel Bacteriocin from Lactobacillus plantarum. Int. J. Curr. Microbiol. App. Sci., 3(5): 227-232.

Diez-Gonzalez, F. 2007. Applications of bacteriocins in livestock. Curr. Issues Intestinal Microbiol., 8: 15-24.

Gaamouche, S., A. Arakrak, M. Bakkali and A. Laglaoui. 2014. Antimicrobial activity of lactic acid bacteria and bacteriocins isolated from a traditional brine table olives against pathogenic bacteria. Int. J. Curr. Microbiol. App. Sci., Volume 3 -11, 657-666.

Gratia, A. 1925. Sur un remarquable example d'antagonisme entre deux souches de colibacille. Compt. Rend. Soc. Biol., 93: 1040-1041.

Gratia, J.P. 2000. "André Gratia: a forerunner in microbial and viral genetics". Genetics, 156(2): 471-6. PMC 1461273.

Jack, R.W., Tagg, J.R., Ray, B. 1995. Bacteriocins of Gram-postive bacteria. Microbiol. Rev., 59: 171-200.

Law, A. 2005. www.hoovers.com.
Malini Maria, Savitha Janakiraman. 2012. Detection of heat stable bacteriocin from Lactobacillus acidophilus NCIM5426 by liquid chromatography/ mass spectrometry. Indian J. Sci. Technol., Vol. 5 No. 3.

NCCLS. 1997. Performance Standards for Antimicrobial Disk Susceptibility Tests: Approved Standard M2-A7. National Committee for Clinical Laboratory Standards, Wayne, PA, USA.PMID 11014798.

Ray, B. 1993. Sublethal injury, bacteriocins and food microbiology. ASM News, 59: 285-291.

Riley, M.A. 2009. Bacteriocins, Biology, Ecology, and Evolution. Encyclopedia of Microbiology. Moselio Schaechter (ed), 32-44.

Sahl, H.G., Bierbaum, G. 1998. Lantibiotics: biological activities of uniquely modified peptides from Gram-positive bacteria. Annual Rev. Microbiol., 52: 41-79.

Sahu, M.K., N.S., Swarnakumar, K., Sivakumar, T., Thangaradjou and L. Kannan. 2008. Probiotics in aquaculture: Importance and future perspectives. Industrial J. Microbiol., 48: 299-308.

Vaughan, E.E., C. Daly, G.F. Fitzgerald. 1992. Identification and characterization of helveticin V-1829, a bacteriocin produced by Lactobacillus helveticus 1829. J. Appl. Bacteriol., 73: 299-308.

\section{How to cite this article:}

Ranganath, S.B., and Sharmila, T. 2016. Isolation and Identification of a Bacteriocinic Substance Producing Bacteria from Various Sources. Int.J.Curr.Microbiol.App.Sci. 5(6): 655664. doi: http://dx.doi.org/10.20546/ijcmas.2016.506.071 